\title{
Correction to: Diet and inflammatory bowel disease: The Asian Working Group guidelines
}

Ajit Sood $^{1} \cdot$ Vineet Ahuja $^{2} \cdot$ Saurabh Kedia ${ }^{2} \cdot$ Vandana Midha $^{3} \cdot$ Ramit Mahajan $^{1} \cdot$ Varun Mehta $^{1} \cdot$ Ritu Sudhakar $^{4}$. Arshdeep Singh ${ }^{1}$ - Ajay Kumar ${ }^{5} \cdot$ Amarender Singh Puri $^{6} \cdot$ Bailuru Vishwanath Tantry $^{7} \cdot$ Babu Ram Thapa $^{8}$. Bhabhadev Goswami ${ }^{9}$. Banchha Nidhi Behera ${ }^{10}$. Byong Duk Ye ${ }^{11}$. Deepak Bansal ${ }^{12}$. Devendra Desai ${ }^{13}$. Ganesh Pai ${ }^{14}$. Ghulam Nabi Yattoo ${ }^{15}$. Govind Makharia ${ }^{2}$. Hasitha Srimal Wijewantha ${ }^{16}$. Jayanthi Venkataraman ${ }^{17}$. K. T. Shenoy ${ }^{18}$. Manisha Dwivedi ${ }^{19}$ - Manoj Kumar Sahu ${ }^{20}$. Meenakshi Bajaj ${ }^{21}$ - Murdani Abdullah ${ }^{22}$. Namrata Singh ${ }^{23} \cdot$ Neelanjana Singh ${ }^{24} \cdot$ Philip Abraham $^{25} \cdot$ Rajiv Khosla $^{26} \cdot$ Rakesh Tandon $^{27} \cdot$ S. P. Misra ${ }^{28} \cdot$ Sandeep Nijhawan ${ }^{29}$. Saroj Kant Sinha ${ }^{30}$. Sawan Bopana ${ }^{31}$. Sheela Krishnaswamy ${ }^{32}$. Shilpa Joshi ${ }^{33}$. Shivram Prasad Singh ${ }^{34}$. Shobna Bhatia ${ }^{35}$. Sudhir Gupta ${ }^{36} \cdot$ Sumit Bhatia $^{37}$. Uday Chand Ghoshal ${ }^{38}$

Published online: 28 January 2020

(C) Indian Society of Gastroenterology 2020

Correction to: Indian Journal of Gastroenterology

$$
\text { 38(3):220-246 }
$$

https://doi.org/10.1007/s12664-019-00976-1

The recommendation 31 which recommends "VSL\#3®", refers only to the product used in the cited literature and is independent from the present product labeling. This product is now known by the generic name "De Simone Formulation".

The authors would like to apologise for any inconvenience caused.

The online version of the original article can be found at https://oi.org/ 10.1007/s12664-019-00976-1

Ajit Sood

ajitsood10@gmail.com

Extended author information available on the last page of the article 


\section{Affiliations}

Ajit Sood ${ }^{1} \cdot$ Vineet Ahuja $^{2} \cdot$ Saurabh Kedia ${ }^{2} \cdot$ Vandana Midha $^{3} \cdot$ Ramit Mahajan $^{1} \cdot$ Varun Mehta $^{1} \cdot$ Ritu Sudhakar $^{4}$. Arshdeep Singh ${ }^{1} \cdot$ Ajay Kumar ${ }^{5} \cdot$ Amarender Singh Puri $^{6} \cdot$ Bailuru Vishwanath Tantry $^{7}$ • Babu Ram Thapa ${ }^{8}$. Bhabhadev Goswami ${ }^{9}$. Banchha Nidhi Behera ${ }^{10}$. Byong Duk Ye ${ }^{11} \cdot$ Deepak Bansal ${ }^{12} \cdot$ Devendra Desai $^{13}$. Ganesh Pai ${ }^{14}$. Ghulam Nabi Yattoo ${ }^{15}$. Govind Makharia ${ }^{2} \cdot$ Hasitha Srimal Wijewantha ${ }^{16}$. Jayanthi Venkataraman ${ }^{17}$. K. T. Shenoy ${ }^{18}$. Manisha Dwivedi ${ }^{19}$. Manoj Kumar Sahu ${ }^{20}$. Meenakshi Bajaj ${ }^{21}$ - Murdani Abdullah ${ }^{22}$. Namrata Singh ${ }^{23}$. Neelanjana Singh ${ }^{24} \cdot$ Philip Abraham $^{25} \cdot$ Rajiv Khosla $^{26} \cdot$ Rakesh Tandon $^{27}$ - S. P. Misra ${ }^{28}$. Sandeep Nijhawan ${ }^{29}$. Saroj Kant Sinha ${ }^{30}$. Sawan Bopana ${ }^{31}$. Sheela Krishnaswamy ${ }^{32}$ - Shilpa Joshi ${ }^{33}$. Shivram Prasad Singh ${ }^{34}$. Shobna Bhatia ${ }^{35}$. Sudhir Gupta ${ }^{36}$. Sumit Bhatia ${ }^{37}$. Uday Chand Ghoshal ${ }^{38}$

1 Department of Gastroenterology, Dayanand Medical College and Hospital, Ludhiana 141 001, India

2 Department of Gastroenterology, All India Institute of Medical Sciences, New Delhi 110 023, India

3 Department of Internal Medicine, Dayanand Medical College and Hospital, Ludhiana 141 001, India

4 Department of Dietetics, Dayanand Medical College and Hospital, Ludhiana 141 001, India

5 BLK Super Speciality Hospital, New Delhi 110 005, India

6 Department of Gastroenterology, GB Pant Hospital, New Delhi 110 002, India

7 Department of Gastroenterology, Kasturba Medical College, Mangalore 575 001, India

8 Department of Gastroenterology Postgraduate Institute of Medical Education and Research, Chandigarh 160 012, India

9 Department of Gastroenterology, Gauhati Medical College, Guwahati 781 032, India

10 Department of Dietetics, Postgraduate Institute of Medical Education and Research, Chandigarh 160 012, India

11 Department of Gastroenterology, Asan Medical Center, Seoul, South Korea

12 Consultant Gastroenterology, Bathinda 151 001, India

13 P. D. Hinduja Hospital and Medical Research Centre, Mumbai 400 016, India

14 Department of Gastroenterology, Kasturba Medical College, Manipal 576 104, India

15 Sher-I-Kashmir Institute of Medical Sciences, Srinagar 190 011, India

16 Colombo North Teaching Hospital, Ragama, Sri Lanka

17 Dr Kamakshi Memorial Hospital, Chennai 600 100, India

18 Department of Gastroenterology, Sree Gokulum Medical College and Research Foundation, Trivandrum 695 011, India

19 Department of Gastroenterology, Moti Lal Nehru Medical College, Allahabad 211 001, India
20 Department of Gastroenterology, IMS and Sum Hospital, Bhubaneswar 756 001, India

21 Dietician, Kilpauk, Chennai 600 010, India

22 Department of Internal Medicine, Faculty of Medicine Universitas Indonesia, Dr. Cipto Mangunkusumo General Hospital, Jakarta, Indonesia

23 Department of Gastroenterology and Human Nutrition, All India Institute of Medical Sciences, New Delhi 110 023, India

24 Dietician, Pushpawati Singhania Research Institute, New Delhi 110 001, India

25 P D Hinduja Hospital and Medical Research Centre, Veer Savarkar Marg, Cadel Road, Mahim, Mumbai 400 016, India

26 Max Super Speciality Hospital, Saket, New Delhi 110 017, India

27 Pushpawati Singhania Research Institute, New Delhi 110 001, India

28 Department of Gastroenterology, Moti Lal Nehru Medical College, Allahabad 211 001, India

29 Department of Gastroenterology, SMS Medical College, Jaipur 302 004, India

30 Department of Gastroenterology, Postgraduate Institute of Medical Education and Research, Chandigarh 160 012, India

31 Fortis Hospital, Vasant Kunj, New Delhi 110 070, India

32 Dietician, Bengaluru 560 001, India

33 Dietician, Mumbai Diet and Health Centre, Mumbai 400 001, India

34 Department of Gastroenterology, Sriram Chandra Bhanj Medical College and Hospital, Cuttack 753 001, India

35 Department of Gastroenterology, King Edward Memorial Hospital, Mumbai 400 012, India

36 Shubham Gastroenterology Centre, Nagpur 440 001, India

37 Consultant Gastroenterology, Medanta The Medicity, Gurgaon 122 001, India

38 Department of Gastroenterology, Sanjay Gandhi Postgraduate Institute of Medical Sciences, Lucknow 226 014, India 